\title{
Transcriptional Regulation of Innate Lymphoid Cell Fate
}

Nicolas Serafini $^{1,2}$, Christian A.J. Vosshenrich ${ }^{1,2}$ and James P. Di Santo ${ }^{1,2,3}$

${ }^{1}$ Innate Immunity Unit, Institut Pasteur, Paris, FRANCE

${ }^{2}$ Inserm U668, Paris, FRANCE

${ }^{3}$ Correspondence to:

James Di Santo

Innate Immunity Unit

Institut Pasteur

25 rue du Docteur Roux

Paris, FRANCE

e-mail: james.di-santo@pasteur.fr

Tel: + 33145688696

Fax: + 33140613510 


\section{ABSTRACT}

Innate lymphoid cells (ILCs) are a recently described family of lymphoid effector cells that have important roles in immune defense, inflammation and tissue remodeling. It has been proposed that ILCs represent 'innate' homologues of differentiated effector T cells and they have been categorized into three groups (namely ILC1, ILC2 and ILC3) based on their expression of cytokines and transcription factors typically associated with $T$ helper $\left(T_{H}\right)-1, T_{H} 2$ and $T_{H}$ 17-type immune responses, respectively. Indeed, remarkable similarity is seen between the specific transcription factors required for the development and diversification of different ILC groups and those that drive effector T cell differentiation. The recent identification of dedicated ILC precursors has provided a view of the mechanisms that control this first essential stage of ILC development. Here, we discuss the transcriptional mechanisms that regulate ILC development and diversification into distinct effector subsets with key roles in immunity and tissue homeostasis. We further caution the current distinction between 'helper' versus 'killer' subsets in the evolving area of ILC nomenclature.

\section{KEY POINTS}

- Three distinct ILC groups have been categorized with unique and overlapping functions in immunity

- Several committed ILC-restricted progenitor subsets have been discovered and the transcriptional programmes that drive their generation have been revealed

- A complex repertoire of transcription factors has been shown to control ILC diversification and maturation

- ILC subsets within tissues are maintained and tuned through a set of environmental signals that balance cellular homeostasis and functional plasticity 


\section{INTRODUCTION}

The innate lymphoid cells (ILCs) are a novel lymphocyte subfamily that is distinguished from the $T$ cells and $B$ cells of the adaptive immune system by their lack of clonotypic antigen receptors and by their independence from the DNA rearrangement machinery (they develop in the absence of Rag1 or Rag2 genes). ILCs have morphological characteristics of lymphoid cells and, like T and B cells, develop from common lymphoid progenitors (CLPs) that express the interleukin (IL)-7 receptor (IL7R $\alpha$; CD127). Moreover, mature ILCs express the characteristic surface receptors and effector molecules of differentiated $T$ cell subsets under the control of specific transcription factors underlining their similarities to their adaptive lymphoid counterparts. Nevertheless, while adaptive immune responses normally require days to weeks in order to fully differentiate, ILCs are early effectors of immunity, responding within hours after activation. As such, ILCs and T cells can be viewed as providing coherent effector functions that differ mostly with regards to kinetics.

ILCs have been categorized into three major groups based on their phenotypic and functional similarities to $T_{H}$ cells (reviewed in ${ }^{1,2}$; Figure 1). Group 1 ILCs (or ILC1s) produce ' $T$ helper $\left(T_{H}\right)-1$ type' cytokines (such as interferon- $\gamma$; IFN- $\gamma$ ) and include natural killer (NK) cells as well as other IFN- $\gamma$ producing ILCs. Group 2 ILCs (or ILC2s) produce $\mathrm{T}_{\mathrm{H}} 2$-type cytokines (such as IL-5 and IL-13) and include natural helper cells, nuocytes and innate helper 2 cells. Group 3 ILCs (or ILC3s) comprise several phenotypically distinct subsets, including lymphoid tissue-inducer (LTi) cells and mucosal natural cytotoxicity receptor (NCR)-bearing cells that produce $T_{H}$ 17-type cytokines, namely IL-17A and IL-22. While this classification privileges certain 'signature' features of ILCs, it should be emphasized that the range of functional capacities of different ILC groups is not fully appreciated, leaving room for further refinement (and possible redefinition) of the ILC nomenclature. Still, the current ILC grouping is supported by the remarkable degree of conservation between ILCs in mice and humans.

Our knowledge of the signals and mechanisms that regulate ILC differentiation from haematopoietic precursors is still in its infancy. In this Review, we will examine the transcription factor pathways regulating ILC development, differentiation and stability. A better understanding of the critical stages and regulators of ILC differentiation could eventually lead to new experimental or therapeutic approaches for manipulating the immune system in the early stages of pathogenic infection or during inflammatory disease.

\section{ILC subsets}

ILC1: including NK cells and other IFN $\gamma$ producing innate cells

The first member of the ILC family was discovered about 40 years ago, when natural killer (NK) cells were characterized in mice and humans (reviewed in ${ }^{3,4}$ ). Functionally, NK cells are able to eliminate infected or 'stressed' target cells via release of cytotoxic granules or via cell surface associated FAS ligand or TNF-related apoptosis-inducing ligand (TRAIL) mechanisms. As such, NK cells are crucial players in tumour immunosurveillance. In addition, NK cells possess a potent cytokine production potential, notably for $\mathrm{T}_{H} 1$ cytokines, including IFN- $\gamma$ and tumour necrosis factor $\alpha$ (TNF), which is especially important in the context of infections by intracellular bacteria. Interestingly, requirements for 
NK cell cytotoxicity and cytokine production depend on the disease context; in some cases, NK cell 'killing' is redundant for NK cell-mediated immune protection in certain organs ${ }^{5}$.

Although both mouse and human NK cells uniformly express the NCR NKp46, there is substantial heterogeneity within these populations in both species. Human NK cells can be divided into $\mathrm{CD}_{56}{ }^{+} \mathrm{CD} 16^{+}$and $\mathrm{CD} 56^{\text {bright }} \mathrm{CD} 16^{-}$subsets $^{6}$. $\mathrm{CD}^{2} 6^{+} \mathrm{CD} 16^{+} \mathrm{NK}$ cells are the predominant $\mathrm{NK}$ cell subset in the spleen and non-lymphoid tissues (such as the lungs and liver), whereas CD56 ${ }^{\text {bright }} \mathrm{NK}$ cells are enriched in lymphoid organs. Both $\mathrm{CD} 56^{+} \mathrm{CD} 16^{+}$and $\mathrm{CD} 56^{\text {bright }} \mathrm{NK}$ cell subsets express the transcription factor eomesodermin (EOMES), are cytotoxic and produce cytokines (Figure 1).

Heterogeneous NK cell subsets in the mouse include CD27 $7^{+} \mathrm{CD} 11 \mathrm{~b}^{-}$and $\mathrm{CD} 27^{-} \mathrm{CD} 11 \mathrm{~b}^{+} \mathrm{NK}$ cells that are found throughout the body (Figure 1). Specialized NKp46 ${ }^{+} \mathrm{NK} 1.1^{+}$cell subsets with distinct functional properties have been described in the thymus, liver, pancreas and uterus (reviewed $\mathrm{in}^{3,4}$ ) and more recently in mucosal sites ${ }^{7,8}$. These cells share the expression of NKp46, NK1.1, and NKG2D with conventional NK cells; they are T-bet ${ }^{+}$and produce $T_{H} 1$-type cytokines. In contrast to NK cells, these NKp46 ${ }^{+} \mathrm{NK} 1.1^{+}$cells lack expression of MHC-specific inhibitory receptors, VLA2 (CD49b, DX5) and Eomes, rendering them devoid of perforin and granzyme-mediated cytotoxic granules. Instead, these cells express the $\alpha 1 \beta 1$ integrin (also known as VLA1 or CD49a/CD18) and Tnfsf10 (encoding TRAIL), which allows them to eliminate target cells via a granzyme-independent pathway ${ }^{9}$. Thus, two T-bet ${ }^{+}$IFN $\gamma$-producing ILC1 subsets exist in mice: one that expresses Eomes and harbors cytotoxic granules, and one that lacks Eomes expression but is TRAIL ${ }^{+}$. Studies using Eomes-reporter mice suggest that these two ILC1 subsets have stable phenotypic and functional characteristics ${ }^{8,10}$.

In humans, a liver-resident subset of $\mathrm{CD} 49 \mathrm{a}^{+} \mathrm{CD} 56^{\text {bright }} \mathrm{NK}$ cells also exists that expresses Tbet but lacks EOMES ${ }^{11}$. In addition, an unusual tonsillar ILC1 subset has been identified ${ }^{12}$. These CD $161^{+} \mathrm{T}^{- \text {bet }^{+}}$cells produce abundant IFN $\gamma$ but lack most classical NK cell markers (namely NKp46, CD56, CD16 and CD94) and do not express perforin or granzyme B. It is not known whether these human $\mathrm{CD} 161^{+} \mathrm{T}_{\text {-bet }}{ }^{+}$cells express TRAIL. Interestingly, this novel human ILC1 subset was enriched in mucosal tissues and expanded following intestinal inflammation, suggesting a role for these cells in mucosal pathologies ${ }^{12}$.

How should we classify these different IFN $\gamma$-producing ILCs? Clearly all of these cells are all ILC1 since they are T-bet ${ }^{+}$and promptly produce type 1 cytokines. Recently, the case has been made to consider a separate 'killer' ILC lineage (including most conventional NK cells) that is independent from 'helper' ILCs and especially distinct from ILC1s ${ }^{8}$. We would argue against this nomenclature as NK cells and other ILCs have more common features than differences, especially with regards to 'helper' functions (BOX 1). We instead propose that two ILC1 subsets should be distinguished: EOMES $^{+}$ILC1 (that includes NK cells) and EOMES ${ }^{-}$ILC1 (Figure 1). The developmental pathways and transcription factors that condition generation of these different ILC1 subsets will be addressed in detail below.

ILC2: including natural helper cells, nuocytes, innate helper 2 cells

Several groups have described ILC subsets that abundantly produce type 2 cytokines, including IL-5, IL-9, and IL-13 ${ }^{13-15}$. These cell populations were initially denoted 'natural helper cells', 'nuocytes', or 
'innate helper 2 cells ${ }^{, 13-15}$ but more recently were designated as group 2 ILC (or ILC2s) s, $^{1,2}$ ILC2s express the IL-25 receptor (also known as IL-17RB), IL-33 receptor (also known as T1/ST2 or IL1RL1) and the receptor for thymic stromal cell lymphopoietin (TSLP) (Figure 1). As such, they are poised to respond rapidly to IL-25, IL-33 and TSLP produced by epithelial cells and haematopoietic cells during inflammation or infection ${ }^{13-16,16-22}$. In mice, ILC2s are distributed in the blood, in lymphoid tissues (such as spleen and bone marrow), in non-lymphoid tissues (including the liver, kidney, visceral adipose) and in mucosal tissues (such as the lung, intestine and fat-associated mesenteric lymphoid clusters) $)^{13,15,22-24}$. In addition to producing IL-5 and IL-13, ILC2 can also produce IL-2, IL-6, granulocyte-macrophage colony-stimulating factor (GM-CSF) and the epidermal growth factor related protein amphiregulin ${ }^{13,14,16,21,24}$. Consequently, ILC2s promote eosinophil recruitment and homeostasis (via IL-5 production) ${ }^{22}$, mast cell and T cell proliferation (via IL-9 production) ${ }^{25}$ and they drive mucus production by goblet cells (via the secretion of IL-13) ${ }^{13-15}$. Interestingly, ILC2-derived IL-9 may promote ILC2 homeostasis in an autocrine manner, providing a feed-forward loop for amplifying ILC2 responses ${ }^{25}$. ILC2s are activated during parasitic worm infection ${ }^{13-15}$, viral infection ${ }^{20,21}$, during allergic reactions ${ }^{26}$ and also associated with chronic human diseases, such as atopic dermatitis ${ }^{27}$, pulmonary ${ }^{28}$ and hepatic fibrosis ${ }^{29}$.

ILC2s have also been characterized in human fetal and adult tissues and in the circulation. Human ILC2s express $T_{H} 2$ chemo-attractant receptor (CRTH2 or CD294) that serves as a high affinity receptor for prostaglandin D2 and is also expressed by $\mathrm{T}_{\mathrm{H}} 2$ cells. Both mouse and human ILC2s express high levels of the transcription factor GATA $3^{23,30-33}$, which regulates ILC2 development and is critical for type 2 cytokine secretion ${ }^{21,23,31,32,34}$. Several other transcription factors (including Notch, TCF1 and ROR $\alpha$ ) are critical for ILC2 generation, as will be discussed below. Increased ILC2 numbers and activation have been demonstrated in human inflammatory syndromes, including allergic asthma and airway hyper-reactivity ${ }^{35}$. Recently, ILC2s have been shown in both mice and humans to regulate metabolic tone through 'beiging' of adipose tissue via secretion of methionine-enkephalin peptides ${ }^{36,37}$.

To what degree ILC2s are functionally heterogeneous remains unclear. Subsets of ILC2s in the bone marrow, lymphoid tissues or lungs of humans and mice differentially express CCR6, CCR9, CD117, CD25, KLRG1 and/or SCA1 (reviewed in ${ }^{38}$ ). Whether these subsets represent immature ILC2 or mature cells under different states of activation remains unclear. Following activation, most ILC2s secrete both IL-5 and IL-13. In contrast, whether other soluble factors produced by ILC2 are concomitantly expressed or segregate into ILC2 subsets with distinct functional capacities has not been shown. In a similar fashion, the role of tissue environments for imprinting functional differences on ILC2s is unclear.

\section{ILC3: including LTi cells and NCR $R^{+}$IL-22-producing cells}

$\mathrm{LTi}$ cells in mice were first described as $\mathrm{CD} 3^{-} \mathrm{CD} 4^{+}$lymphocytes that promote lymphotoxin- $\alpha / \beta$ and RANKL-mediated lymphoid tissue organogenesis during fetal life (reviewed in ${ }^{39}$ ). In response to microbial signals received after birth, LTi cells transform cryptopatches ${ }^{40}$ into isolated lymphoid follicles (ILFs) that allow for the generation of IgA-producing plasma cells ${ }^{41,42}$. LTi cells were 
subsequently shown to play a role in immune defense against infection during adult life through production of the inflammatory cytokines IL-17A and IL-22 $2^{43,44}$. LTi cells acquire these diverse functions due to their expression of the transcription factor RAR-related orphan receptor gamma $(R O R \gamma t)$, which is essential for their generation ${ }^{43,45,46}$.

More recently, other RORyt-dependent ILC subsets were identified, such as $\mathrm{NCR}^{+}$cells (NKp46 in mouse; NKp44 in humans) that lack cytotoxicity and IFN $\gamma$ production (thereby distinguishing them from ILC1) but having potent IL-22 production capacity in response to stimulation by IL-23 and/or IL-1 $\beta$. These cells were initially denoted as 'NK22', 'NCR22', 'NK-LTi' and 'ILC22'47-49. Considering their dependence on ROR $\gamma$ t and their functional profiles (IL-17A, IL-22, LTs), LTi cells and NCR ROR $\gamma^{+}$were categorized together as group 3 ILC (or ILC3; Figure 1) that can be considered as innate versions of $T_{H} 17$ cells or ' $T_{H} 22$ ' cells ${ }^{1,2}$. In both mice and humans, NCR ILC3 are enriched in mucosal sites (such as the intestinal lamina propria and Peyer's patches) but are rare in other lymphoid tissues, including the spleen and peripheral lymph nodes, at steady-state ${ }^{47,48,50-52}$. Gut commensal bacteria are involved in homeostasis of $\mathrm{NKp}_{4} 6^{+} \mathrm{ILC} 3$ in mice ${ }^{47,48}$, although the mechanism by which this is regulated is unclear.

In addition, heterogeneous $\mathrm{CD}^{-} \mathrm{NCR}^{-}$ILC3 subsets have been described that express combinations of CCR6, T-bet and MHC class II and produce IL-17A, IL-22 and IFN $\gamma^{50,51,53}$. Some of these ROR $\mathrm{t}^{+}$ILCs appear pathogenic and are associated with colonic inflammation in mouse models (Helicobacter hepaticus infection and anti-CD40-mediated inflammation) ${ }^{50}$. In contrast, a fraction of ILC3 constitutively express MHC class II molecules that appear to have a role in maintaining intestinal $\mathrm{T}$ cell homeostasis and activation ${ }^{53}$. Thus, a remarkable functional diversity (either protective or pathogenic) within distinct ROR $\mathrm{t}^{+}$ILC3 subsets exists. Understanding the role for distinct ILC3 subsets in immune defense and tissue homeostasis is challenging but could provide important information that could translate to better disease prevention or treatment. Several inflammatory diseases (inflammatory bowel disease or IBD, psoriasis, rheumatoid arthritis) in humans are associated with excessive IL-23 stimulation leading to high levels of IL-17 and/or IL-22 ${ }^{54}$. In humans, cytokine-producing ILC3 are found in intestinal tissues ${ }^{55,56}$, lymph nodes ${ }^{57}$, tonsil, Peyer's patches and appendix ${ }^{58}$ and in patients with IBD, the homeostasis of IL-22 and IL-17 producing ILC3 populations appear disturbed ${ }^{59}$. However, more work is required in order to fully understand ILC3 subset complexity and to firmly establish roles for these subsets in healthy and diseased human tissues.

Other 'innate' effector cells have been described that share some functional characteristics with ILCs, although these cell types also have some clear developmental differences (see BOX 2). Are these ILCs? What criteria should be used to include immune effectors in the ILC family? The characterization of distinct groups of ILCs and heterogeneous subsets within individual ILC groups underscores the remarkable degree of ILC diversity. This diversity, however, raises new questions: What are the developmental pathways that guide differentiation of ILC subsets? How is ILC diversification achieved? Where does ILC differentiation occur? Are ILC subsets functionally fixed or plastic? We will address these questions in the subsequent sections.

\section{ILC development}

6 TAPEZ LE TITRE DU DOCUMENT 
Within the haematopoietic system, multi-potent, self-renewing hematopoietic stem cells (HSCs) provide the cellular substrate for the generation of all hematopoietic lineages (ie: erythroid, megakaryocytoid, myeloid and lymphoid cells). Hematopoiesis is a continuous process, characterized by the progressive loss of cell fate potentials. During lymphopoiesis, erythroid, megakaryocytoid and myeloid potentials are shed, resulting in the generation of common lymphoid progenitors (CLPs) that are restricted to the lymphoid fate. Several transcription factors are involved in CLP generation (including Ikaros, E2A, PU.1 and GFI1) and appear to prime the lymphoid programme even at the earliest stages (HSC) of this process ${ }^{60}$. These transcription factors concomitantly activate the lymphoid-restricted gene programmes (including expression of the IL-7R $\alpha$ and the recombinase activating genes - Rag1 and Rag2) and strongly repress the myeloid programme. The result is a cell population that has restricted potential for B cell, T cell and ILC fates. We propose that ILC development be considered in three main stages, namely; 'stage one', the generation of common ILC precursors; 'stage 2', the diversification of ILC precursors into each of the three main ILC groups; and 'stage 3', ILC maintenance and regulation in peripheral tissues (Figure 2).

\section{Stage 1: Generation of Common ILC Precursors}

Lymphoid-restricted precursors for innate or adaptive immunity

The committed precursors that give rise to cells of the adaptive immune system have been well characterized: they include pro-B cells within the bone marrow and pro-T cells in the thymus that express transcriptional programmes of $B$ and $T$ cell 'identity' (reviewed in ${ }^{61}$ ). These precursors are restricted to the $\mathrm{B}$ or $\mathrm{T}$ cell fate; under normal circumstances, no other lymphoid (or non-lymphoid) potentials are available to these cells. For the ILC family, committed precursors have also been identified that can give rise to multiple ILC subsets. Previous studies have identified rare lineagenegative cells in fetal liver, fetal gut and adult bone marrow that express CD127 (IL-7R $\alpha$ ) and the $\alpha 4 \beta 7$ integrin and have lost B or T cell potential, but can generate NK cells, dendritic cells and LTi cells $^{62}$. By excluding all differentiated lymphocyte subsets, rare populations remain that have the characteristics of committed ILC precursors. Several groups recently identified these cells using mice that report on Id2, Zbtb16 (encoding PLZF; promyelocytic leukemia zinc finger) or Cxcr6 expression $^{8,63-65}$. These precursors have been denoted 'CHILP' (for common helper ILC precursor), $\mathrm{PLZF}^{+}$ILC precursors and $\alpha \mathrm{LP}$ (for a lymphoid precursors): they will be referred to as common ILC precursors (CILCPs) here for simplicity (Figure 2).

CILCPs lack B and T cell potential, have no myeloid potential and give rise to the three main ILC groups after adoptive transfer in vivo. Moreover, in vitro, CILCPs can give rise at the single cell level to multiple ILC groups and in the case of CXCR6 ${ }^{+}$CILCPs, can generate EOMES ${ }^{+}$ILC1 as well as EOMES ILC1, ILC2 and ILC3 at the single cell level ${ }^{65}$. These studies provide strong evidence that committed ILC precursors exist in mice, although the functional characteristics of these CILCP populations are somewhat distinct, suggesting further complexity. For example, Id $2^{+}$and $\mathrm{PLZF}^{+} \mathrm{ILC}$ precursors appear to lack potential for EOMES ${ }^{+}$ILC1s (that is, NK cells), whereas $\mathrm{PLZF}^{+}$precursors also failed to generate LTi cells of the ILC3 subset in vivo or in vitro ${ }^{8,64}$. These observations suggest a model whereby CILCPs may progressively lose the capacity to generate particular ILC subsets as they differentiate: in this scenario, CXCR6 ${ }^{+}$CILCPs would represent the most 'immature' CILCPs 
whereas $\mathrm{PLZF}^{+}$CILCP would include more 'mature' precursors (Figure 3a). The fact that PLZF expression can be induced in $\mathrm{CD} 127^{+} \alpha 4 \beta 7^{+}$cells by Notch signaling ${ }^{64}$ is consistent with this model. Alternatively, these different CILCPs may indicate unique pathways of ILC development due to transcription factors requirements (Figure 3b; but also see BOX1). Studies assessing the precursorproduct relationships of these different CILCPs with direct side-by-side comparison for in vitro and in vivo potential should help resolve these issues.

Along these lines, an additional discussion concerning committed NK precursors (NKPs) is warranted. While the existence of NKPs has been known for some time ${ }^{66}$, a more recent characterization helped to further define NKP generation from $\mathrm{CLPs}^{67,68}$. A population of cells that are lineage ${ }^{-} \mathrm{CD} 127^{+} \mathrm{CD} 27^{+} \mathrm{CD} 244^{+} \mathrm{FIt}^{-}$and variable for the expression of $\mathrm{CD} 122$ (the IL-2RR) is has been described that lies upstream of NKPs in the adult mouse bone marrow ${ }^{68}$. This pre-NKP population lacks $B, T$ and myeloid cell potential but robustly generates NK cells after adoptive transfer in vivo. Although these observations clearly support the existence of NK cell-restricted precursors, the ability of pre-NKP cells to generate other ILC subsets (EOMES ${ }^{-}$ILC1, ILC2, ILC3) is not known. Understanding the relationship of pre-NKPs to other CILCPs may help to distinguish between these different models of early ILC precursor development (Figure 3).

\section{Transcription factors that promote common ILC precursor generation}

Several transcription factors have been identified that impact on the generation of multiple ILC subsets, including inhibitor of DNA-binding 2 (ID2), nuclear factor IL-3 induced (NFIL3, also known as E4BP4), and GATA3 (Figure 2). The broad effect of ablation of these transcription factors on ILC homeostasis can in part be explained by their role in promoting emergence of ILC precursors from CLPs. We will briefly summarize how selected transcription factors may orchestrate this process.

ID2. ID2 is a member of the ID family of transcriptional repressors, which broadly regulate cellular differentiation in different organ systems. ID proteins lack a DNA-binding domain and thus do not have the capacity to directly modulate gene transcription. They instead act indirectly via binding to and functionally inactivating a set of transcriptional activators (the so-called ' $E$ ' proteins) that includes E2A (E12/E47), E2-2 and HEB. Within the haematopoietic system, ID2 is expressed in multiple cell lineages and its ablation has diverse effects on the differentiation of myeloid and lymphoid cells (reviewed in ${ }^{69}$ ). An early report described a selective loss of NK cells and lymphoid tissues in ID2-

deficient mice ${ }^{70-72}$. As primary $B$ and $T$ cell development was essentially unperturbed in these mice, a model emerged in which levels of $E$ protein activity would dictate the choice between $B$ and $T$ cells on the one hand, and NK cells on the other hand in early lymphoid precursors. Consistent with this model, early B- and T-lineage development is strongly perturbed in mice deficient in E2A, E2-2 and/or HEB ${ }^{73-}$ 75. Since then, ID2 has been shown to be essential for the development of all known ILC subsets $^{8,46,62,70}$ reinforcing this general principle. Moreover, over-expression of ID proteins inhibits B and $T$ cell development, while strongly promoting ILC (specifically NK cell) generation ${ }^{76}$.

As CLPs express little or no ID2 ${ }^{8,31,64}$, the signals that regulate its expression should impact strongly on the choice of B or T cell versus ILC development by altering the overall E protein activity 
within these precursors. High levels of ID2 would essentially restrict lymphoid precursors to the ILC lineage, a model consistent with the characteristics of ID2 ${ }^{+}$and PLZF ${ }^{+} \mathrm{CILCPs}^{8,64}$. Bone morphogenic proteins (BMPs) trigger the SMAD pathway and appear to directly regulate ID2 transcription in some cell types ${ }^{77}$. In contrast, signals that maintain E protein activity in CLPs would counteract this process. Interestingly, some ILCs can develop in the absence of ID2 if E proteins are also ablated ${ }^{70}$. These observations would suggest that ILC development might represent a default pathway from CLPs.

NFIL3. The transcriptional regulator NFIL3 is broadly expressed in multiple tissues and is involved in multiple developmental processes, including regulation of circadian rhythm ${ }^{78,79}$. NFIL3 can be associated with either activating or repressive functions dependent on its assembly with other cofactors. Within the hematopoietic system, NFIL3 has multiple roles and regulates development and differentiation of several different lineages (reviewed in ${ }^{80}$ ). Within lymphocytes, NFIL3 ablation has a dramatic effect on NK cell development at the pre-NKP stage but NFIL3 expression does not seem to essential in mature NKp46 ${ }^{+}$cells ${ }^{81,82}$. Recently, several groups have described defects in multiple ILC subsets in NFIL3-deficient mice, including loss of IFN $\gamma^{+}$intestinal ILC1s, systemic defects in ILC2s, and reduced numbers of ILC3 resulting in loss of mucosal defense (ILC3) ${ }^{83,84,85}$. The broad impact of NFIL3 loss on several ILC groups suggests a role for this transcription factor in early lymphopoiesis and possibly in the generation of CILCPs. This appears to be the case, as PLZF ${ }^{+}$CILCPs and ID2 ${ }^{+}$ CILCPs express high levels of NFIL3 and are strongly decreased in the absence of NFIL $3^{65,85}$. Thus NFIL3 appears to be an important orchestrator of ILC precursor emergence from CLPs.

As is the case for ID2, CLPs express little if any NFIL3, so signals that regulate NFIL3 expression could impact on B cell, T cell and ILC fate decisions in these early precursors. NFIL3 levels can be regulated by cytokines ${ }^{86}$ and IL-7 can strongly induce NFIL3 expression in CLP and CILCPs ${ }^{85}$. Once expressed, NFIL3 can influence transcriptional profiles although relevant NFIL3 targets in early lymphoid precursors are not clearly identified. One possibility is that NFIL3 represses B or T cell programmes, thereby promoting ILC fate. ID2 would be a logical candidate for NFIL3-mediated activation, although ID2 levels are apparently not altered in NFIL3-deficient NK cells ${ }^{87}$ or NFIL3deficient ILC2 ${ }^{85}$. Still, NFIL3 could control ID2 at an earlier stage, and evidence for activated dimethylated H3K4 in the NFIL3-binding region of ID2 in CILCPs has recently been demonstrated ${ }^{85}$. Other targets that may be activated by NFIL3 include the transcription factors thymocyte selectionassociated high mobility group box protein (TOX) and EOMES ${ }^{65,88}$, although no clear evidence has been provided that these transcription factors broadly impact on ILC homeostasis. More work is required to understand how NFIL3 expression is controlled and the relevant NFIL3 targets in CLP and ILC precursors.

GATA3: The transcription factor GATA binding protein 3 (GATA3) has broad roles in $\mathrm{T}$ cell differentiation, allowing for the generation of the earliest $T$ cell progenitors, promoting several stages of thymocyte differentiation and later as an essential driver of $T$ helper $2\left(\mathrm{~T}_{\mathrm{H}} 2\right)$ cell polarization (reviewed in ${ }^{89}$ ). As expected, GATA3 plays a critical role throughout ILC2 differentiation to ensure IL-5 and IL-13 expression ${ }^{23,31,32}$ (see more below) but somewhat surprisingly is also required for 
development of several ILC1 and ILC3 subsets $^{32,90,91,8}$. In the absence of GATA3, lineage CD127 ${ }^{+}$ cells fail to develop in the fetal liver and adult bone marrow, and mature intestinal EOMES ${ }^{-}$ILC1s as well as all ILC3 subsets (CD4 ${ }^{+} \mathrm{LTi}$ cells, $\mathrm{NCR}^{+}$cells) are missing ${ }^{8,91}$. As such, GATA3 has a broad role in ILC development. It has been proposed that GATA3 segregates 'helper' from 'killer' ILC lineages ${ }^{9}$ (see also BOX 1), because the development of splenic CD127 EOMES ${ }^{+}$ILC1s (NK cells) proceeds in the absence of GATA $3^{92}$. Still, conventional NK cell development is impaired in the absence of GATA3 and some EOMES ${ }^{+}$ILC1 subsets are completely GATA3-dependent, including CD127 thymic NK cells $^{90}$. These observations argue against a simple relationship between GATA-3 and 'killer' and 'helper' ILC subtypes. Alternatively, since GATA3 regulates CD127 expression in early ILC progenitors $^{91}$, the overall effects of GATA3 deletion on ILC development may be explained by lack of IL-7 signals. An in-depth characterization of CILCPs in GATA3-deficient mice may provide answers.

Zbtb16. The Zbtb16 gene encodes the transcription factor PLZF that has critical roles in the differentiation of T cell subsets, especially CD1d-reactive invariant NKT cells (reviewed in ${ }^{93}$ ). Recently, the role for PLZF in ILC differentiation was demonstrated with Zbtb16 ${ }^{\text {CreGFP }}$ mice ${ }^{64}$. As indicated above, $\mathrm{PLZF}^{+}$fetal liver and bone marrow precursors were shown to include committed ILC precursors. However, Zbtb16-deficient mice do not demonstrate broad defects in ILC development, but rather have selective and partial defects in ILC1 and ILC2 development ${ }^{64}$. It is therefore unlikely that PLZF expression is required for generation of CILCP from CLP, although the molecular targets of Zbtb16 at this stage remain unknown.

\section{Stage 2: Diversification of Three Main ILC groups}

Parallels between ILC and TH cell differentiation

The second stage of ILC differentiation involves the diversification of different ILC groups from committed CILCPs (Figure 2). While strong evidence indicates that different transcription factors regulate this process (as described below), the signals that initiate ILC diversification are poorly defined. Considering the strong parallels that mature ILC subsets share with differentiated T cell subsets, one model for ILC differentiation draws heavily upon the current knowledge of $T_{H}$ cell diversification from naive $T$ cells. This process generates $T_{H}$ cells that express 'signature' transcription factors and cytokines; an analogous process could regulate the differentiation of ILC groups (Tbet/IFN $\gamma$ in ILC1s; GATA3/IL-5 and IL-13 in ILC2; ROR $\gamma$ /IL-17 and IL-22 in ILC3) (see also BOX 3). During $T_{H}$ cell differentiation, several signals are required, including TCR triggering, co-stimulation and soluble factors (these have been referred to as signal 1, signal 2 and signal 3, respectively) (reviewed in ${ }^{94}$ ). TCR signallng activates an ITAM (immunoreceptor tyrosine-based activation motif)-dependent pathway that involves the SYK and ZAP70 tyrosine kinases. Co-stimulation triggers a phosphoinositide 3-kinase pathway that assures robust $\mathrm{T}$ cell proliferation, while cytokines associated with signal 3 provide guidance for $T_{H}$ cell polarization. Are analogous signals involved in ILC differentiation? Do 'naïve' undifferentiated ILCP exist? In short, there is too little data to conclude for or against the ' $T_{H}$ ' model of ILC diversification. It is not known whether ITAM signals are required for ILC development (except for in the case of NK cells, where these signals are not required ${ }^{95}$ ). Similarly, the importance of co-stimulation for ILC diversification is not yet demonstrated. Expression of some 
cytokine receptors can be redundant for the generation of certain ILC subsets (IL-12R for ILC1, IL-33R for ILC2, IL-23R for NKp46 ${ }^{+}$ILC3 $)^{14,47,96}$. Still, whether these factors provide relevant signals at the ILC precursor stage and whether ILCP should be considered as 'innate' versions of naïve T cells is not clear.

Whatever the signals, selective up-regulation of 'signature' transcription factors marks the initial step towards diversification into different ILC groups. In some cases, immature stages that represent steps along the path to mature ILCs have been distinguished. Here, we briefly summarize these ILC group-restricted precursors and explain how selected transcription factors guide their differentiation into distinct mature ILC subsets.

\section{Differentiation of group 1 ILCS}

Transcription factors that influence ILC1 commitment include T-bet, EOMES, ETS1 and TOX ${ }^{10,97-99}$. Tbet (encoded at Tbx21) is the 'signature' transcription factor for mature ILC1s that produce IFN $\gamma$. T-bet binds to and activates the Ifng locus via chromatin remodeling that allows for additional binding of factors that up-regulate Ifng expression, such as RUNX3 ${ }^{100}$. Committed precursors for EOMES ${ }^{+}$ILC1 (NK cells) are well characterized and include NKP subsets that express T-bet and EOMES ${ }^{88}$. Committed precursors for EOMES ILC1s are proposed to include $\mathrm{CD}^{-} 127^{+} \mathrm{NKp} 46^{+} \mathrm{NK} 1.1^{+}$bone marrow cells ${ }^{8}$, but these cells have not been rigorously studied. Inducers of T-bet expression in differentiating $T_{H} 1$ cells include STAT1 and STAT4 that are activated in response to IFN $\gamma$ and IL-12, respectively (reviewed in ${ }^{101}$ ). Whether these soluble factors and their associated STAT pathways are involved in early stages of ILC1 commitment from ILC precursors is not known. EOMES has been proposed as a downstream target of NFIL3 in $\mathrm{NKPs}^{88}$ that might drive early stages and could help to explain the strong defect in NK cell development in NFIL3-deficient mice. However, as these mice have defects in multiple ILC subsets (including EOMES ${ }^{-}$ILC1 $^{8,665,83,84}$ ), it is likely that NFIL3 activates multiple targets beyond EOMES. Reporter mice for EOMES and T-bet will be important tools for identifying ILC1 precursors in future studies.

The transcription factor TOX plays multiple roles during intrathymic $T$ cell selection and differentiation ${ }^{102,103}$. TOX is expressed at low levels in $\mathrm{CLPs}^{65}$ and is also expressed by a subset of

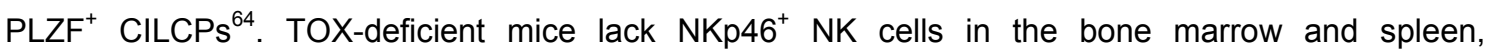
suggesting an important role for this transcription factor in ILC1 development, at least for EOMES ${ }^{+}$ ILC1 $\mathrm{s}^{97}$. The additional impact of TOX-deficiency on ILC3 development (see below) could indicate a role for this transcription factor in a subset of multi-potent ILC precursors. Along these lines, NFIL3 has been proposed to regulate TOX in a subset of CILCP ${ }^{65}$.

ETS1 is a member of the large family of ETS transcription factors (there are 26 members of the ETS family in mice) and has broad roles in development (reviewed in ${ }^{104}$ ). ETS1 deficiency impacts on multiple lymphoid lineages, including B, T and NK cells ${ }^{99,105,106}$. Pre-NKPs are reduced in the absence of ETS1 and transcriptional analyses indicate that ETS1 sustains ID2 and T-bet expression in these progenitors ${ }^{107}$. Upstream regulators of ETS1 in lymphoid precursors are poorly characterized but include cytokines IL-2 and IL-15 in more mature cells. 


\section{Differentiation of group 2 ILCS}

Several transcription factors have been identified that are critical to ILC2 differentiation, including GATA3, GFI1, TCF1 and RORa ${ }^{16,23,31,32,108-110}$. GATA3 is considered a 'signature' transcription factor for mature ILC2s, and cell populations expressing high levels of GATA3 are highly enriched for these cells $^{23,31,32,91}$. GATA3 transcriptional activity activates type 2 cytokine transcription and is required for effector functions in mature ILC2s as well as for their survival ${ }^{34}$. Lower levels of GATA3 are expressed in CILCPs, so GATA3 up-regulation marks the stage of restriction and commitment to the ILC2 lineage. What regulates GATA3 expression at this stage is unknown, but STAT6 may be involved as is the case in differentiating $T_{H} 2$ cells (reviewed in ${ }^{89}$ ). GATA ${ }^{\text {hi }}$ ILC2s can be found in the bone marrow and are proposed to represent ILC2 precursors because of their superior proliferative capacity ${ }^{31}$. Still, bone marrow ILC2s produce type 2 cytokines $^{31}$, so they cannot be considered as functionally immature. IL25 administration expands a KLRG1 ${ }^{+}{ }^{I L}-33 R^{-}$ILC2 population that can further mature into IL-33R ${ }^{+}$ILC2 in vitro ${ }^{111}$. These 'inflammatory' ILC2s (ilLC2) also appear to have the potential to differentiate into ROR $^{+}{ }^{+}$ILC3s ${ }^{111}$ As such, KLRG1 ${ }^{+}$ilLC2 have properties resembling ILC precursors ${ }^{112}$, although their capacity to generate other ILC subsets remains untested. Whether inflammatory ILC2s are an obligate intermediate in ILC2 differentiation under non-inflammatory conditions requires further study.

Notch signaling initiates the $T$ lineage specification, and TCF1 and BCL11B, which are the downstream targets of Notch, are required for $\mathrm{T}$ cell commitment (reviewed $\mathrm{in}^{61}$ ). Although Notch has been shown to have a role in triggering ILC2 development in vitro ${ }^{108,110}$, its role in vivo in this process is still unclear. By contrast, TCF1 is essential for ILC2 development in vivo ${ }^{110,113}$ suggesting close parallels exist between ILC2 development and early T cell development. Considering the close relationship between Notch, Tcf1 and Bcl11b, it is possible that the latter is also involved in ILC2 development.

$\mathrm{ROR} \alpha$ is a transcription factor of the nuclear hormone receptor superfamily. It is required for several developmental processes, most notably for cerebellar Purkinje cell generation as can be seen from the cerebellar defects that occur in staggerer mice, which harbor a deletion within Rora ${ }^{114}$. Rora is highly expressed by multiple ILC subsets ${ }^{108,115}$ but its ablation causes a selective defect in ILC2 numbers, especially in the bone marrow ${ }^{108,115}$. The factors that induce ROR $\alpha$ in the ILC2 lineage are not known but could include GATA3 ${ }^{23}$. The downstream targets of ROR $\alpha$ that promote ILC2 differentiation are similarly undefined.

\section{Differentiation of group 3 ILCs}

The orphan nuclear hormone receptor transcription factor ROR $\gamma$ is essential for the development of group $3 \mathrm{ILCs}^{45,47-49,52}$. Expression of ROR $\gamma$ in early hematopoietic precursors marks those cells that have committed to the ILC3 lineage; these include fetal liver $\alpha 4 \beta 7^{+}$cells in mice ${ }^{46}$ as well as a subset of $\mathrm{CD} 34^{+} \mathrm{CD} 117^{+}$cells in man ${ }^{116}$. ROR $\mathrm{Rt}$ expression in these ILC3 precursors is associated with several hallmarks of mature ILC3s (for example, expression of Ahr, IIIr, II23r, Tox); however, the expression of cytokines such as IL-17 and IL-22 in these cells is rather limited, suggesting that additional signals are required to fully mature these ILC3 precursors ${ }^{51,97,117-121}$. Curiously, these $\mathrm{ROR} \mathrm{ft}^{+}$precursors are extremely rare in adult mouse bone marrow. Together, these observations are 
consistent with the possibility that immature ILC precursors (common or group-restricted) may exit the bone marrow and complete their differentiation within tissues (see below). What drives ROR $\gamma$ t expression at this stage is not fully understood, but transient Notch signals have been proposed to contribute, especially for fetal liver ILC3 precursors ${ }^{46,122}$. Consistent with Notch signalling in ILC3restricted precursors, the transcription factor TCF1 is also important for generating mature ILC3 subsets $^{113}$. Notch signaling is also required for the development of NKp46 $6^{+}{\text {ILC } 3 s^{122,123}}^{\text {. }}$

The transcription factor TOX is also required for the generation of some ILC3 subsets (for instance, LTi cells), whereas NKp46 ${ }^{+}$ILC3s are TOX-independent ${ }^{97}$. How TOX controls the development of LTi cells is unknown, but ID2 appears not to be involved ${ }^{97}$. The aryl hydrocarbon receptor (AHR) is a transcription factor that is involved in the metabolism of xenobiotic compounds and is activated by a diverse set of endogenous ligands. Multiple ILC3 subsets are affected by AHRdeficiency, including LTi cells and NKp46 ${ }^{+}$ILC3 subsets ${ }^{115,117-119}$. By contrast, lymphoid structures are largely intact indicating that fetal ILC3 differentiation may be AHR-independent. It is not known whether ILC3 precursors require AHR expression, but as conditional deletion of Ahr in the ILC3 lineage (using Rorc-Cre) generates similar defects in ILC3s as those seen in AHR-deficient mice, the continued expression of AHR appears necessary for ILC3 homeostasis.

\section{The where and when of ILC differentiation}

ILC development is active during the fetal period (to generate LTi cells involved in lymphoid tissue organogenesis; ${ }^{45,124}$ ) and continues in adulthood. Sites for fetal ILC development include the fetal liver and fetal gut ${ }^{43,46}$. In these different tissue sites, ILC precursors have been identified that include an arginase $1(A R G 1)^{+}$subset that can give rise to multiple ILC lineages in vitro and are associated with developing lymphoid structures in the intestine ${ }^{125}$. ROR $\gamma \mathrm{t}^{+}$ILC3-restricted fetal liver precursors with the potential to generate LTi cells and $\mathrm{NCR}^{+}$ILC3s after migration to the intestine have been described ${ }^{46}$, as well as $C X C R 6^{+} \alpha 4 \beta 7^{+}$fetal liver cells that give rise to NK cells and ILC3s, but not B or T cells ${ }^{65}$. By contrast, little is known about fetal development pathways for other ILC subsets (EOMES ${ }^{-}$ILC1s and ILC2s). Conventional wisdom proposes that the bone marrow is a major site of ILC development in adults that generates mature ILCs that enter the circulation and seed peripheral tissues. For EOMES ${ }^{+}$ ILC1, progenitors and developmental intermediates (including NKP and immature stages) are well characterized ${ }^{3}$. For other ILC subsets, putative precursors for EOMES ${ }^{-}$ILC1s and for ILC2s have been proposed ${ }^{8,31}$. Still, it is not clear to what extent these are true precursors or simply mature ILCs in the 'resting' state.

Beyond the 'bone marrow-centric' view of adult ILC development, it remains possible that circulating CLPs or ILC-restricted precursors (the CILCP subsets described above) could enter tissues allowing for local ILC development. The notion of 'peripheral' NK cell development was previously proposed based on the identification of murine NKPs outside the bone marrow ${ }^{126}$ and the presence of human NKPs in secondary lymphoid tissues ${ }^{127}$. More recently, ILC3-restricted precursors were identified in human tonsils ${ }^{116}$. Although evidence for tissue seeding by CILCPs remains limited, peripheral ILC development may provide the means to promote the generation of ILC subsets within tissues. Environmental signals (for instance, cytokines, cell-associated ligands, microbial products or 
dietary factors?) will likely differ depending on the type of tissue encountered (and perhaps the position of the cell within the tissue) thereby providing a range of potential signals that would influence ILC development and diversification most suitable for the given context.

A final issue related to ILC development concerns the signals that regulate the balance between different ILC subsets in the same tissue. ILC subset distribution differs depending on the tissue and its inflammatory context. In resting lymphoid tissues, ILCs are generally quite rare, with the exception of EOMES ${ }^{+}$ILC1, whereas multiple, diverse ILC subsets are present in mucosal tissues under steady-state conditions and ILC populations expand after infection or inflammation at these sites. Whereas changes in ILC subset distribution is likely to result from altered recruitment and/or proliferation of mature ILCs, it is possible that biased ILC development from tissue-seeding ILC progenitors may also impact on ILC subset representation under these conditions.

\section{Stage 3: ILC maintenance and regulation in peripheral tissues}

Regulation of ILCs in peripheral tissues is critical for optimizing immune defense against infections and also for providing adequate signals for tissue remodeling after insult or inflammation. ILC homeostasis describes the mechanisms that keep the number and diversity of ILCs in the various tissues at an optimal level, and for any given ILC population, involves a combination of processes that impact on ILC generation, circulation, survival/maintenance, proliferation and cell death. A complete discussion of these different processes is out of the scope of this review, but we will touch on a few key aspects that have an impact on peripheral ILC survival and plasticity (Figure 4).

ILC maintenance in tissues - a key role for cytokines

Cytokines of the $\gamma_{c}$-family are essential for the development of all known ILC subsets. The analysis of IL-7-deficient and IL-15-deficient mice, established the role of IL-7 for ILC2 and ILC3 development, and IL-15 for the generation of EOMES ${ }^{+}$and EOMES ILC1s $^{8,126,128,129}$. Moreover, exogenous delivery of these cytokines can expand ILC subsets in vivo ${ }^{130-132}$. It remains unclear at what stage these cytokines exert their effects on ILC homeostasis. As ILC precursors and mature ILCs express the relevant cytokine receptors, it is likely that IL-7 or IL-15 are required at both the earliest progenitor stages but also to maintain the respective cell lineages in the tissues. Recent data derived from

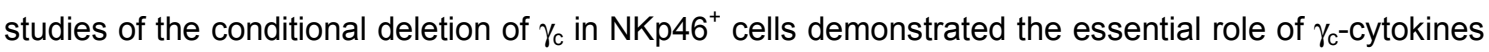
in the homeostasis of EOMES ${ }^{+}$and EOMES ${ }^{-}$ILC1s and NKp46 ${ }^{+}$ILC3s ${ }^{133}$. Additional studies, including ILC transfer experiments and/or cell-type specific targeting of CD122 or CD127 at later stages of ILC differentiation should further define the critical roles for these cytokines in ILC homeostasis. The biologically relevant source of IL-7 or IL-15 for ILC survival remains unclear. IL-15 is required for survival and proliferation of mature NK cells $\mathbf{s}^{134}$ and multiple cell-types contribute via their production of IL-15, including myeloid cells and epithelial cells ${ }^{135}$. Recent data demonstrated that adipocytes also produce IL-15 that helps to maintain not only NK cells but also tissue-resident ILC1s ${ }^{136}$. Reporter mice suggest that diverse stromal cells, epithelial cells and endothelial cells are primary sources of IL-7. As such, ILC homeostasis is likely not to be under the control of a single cellular source, but rather regulated via diverse niches throughout the body. This might explain why systemic IL-7 or IL-15 levels 
are often reported as 'not detected' since they would not measure the cytokine concentration that locally fine-tunes tissue ILC homeostasis.

IL-2 produced by T cells during their activation may also serve to maintain ILC subsets in lymphoid and non-lymphoid tissues. Regulatory T cells bear high-affinity IL-2 receptors that bind IL-2 in tissues; depletion of these cells can alter ILC homeostasis as shown by the increase in ILC1 numbers that occurs in FOXP3-deficient mice ${ }^{137}$. Similarly, IL-2 produced by $\mathrm{T}$ cells has been implicated in ILC2 populations expansion during pulmonary inflammation ${ }^{25}$. The ability of T-cell derived IL-2 as a generic modifier of ILC homeostasis and T regulatory cells to inhibit indirectly this process provides an additional mechanism for control of ILC homeostasis.

\section{ILC lifespan and 'memory'}

Lymphocyte lifespan can vary enormously in different cell types, from several days to a lifetime. Concerning ILC lifespan, cell-transfer experiments and in vivo fate-mapping approaches have estimated the half-life for ILC1 subsets and ILC3 subsets $\left(\mathrm{LTi}, \mathrm{NKp} 46^{+}\right)$to be in the order of 2-3 weeks ${ }^{43,134}$. We have little information on lifespan for other ILC subsets and additional work is needed to answer this question. ILC lifespan may also be conditioned by cellular activation, infection or inflammation that may as well modify effector outputs of ILCs. Along these lines, long-lived NK cells that expand in a ligand-specific fashion can be recovered after MCMV infection: such 'memory' NK cells have enhanced functional properties ${ }^{138}$. Immune responses that activate ILC2 and ILC3 populations are also associated with increases in ILC numbers; it will be interesting to study whether these expanded ILCs have 'memory' properties.

\section{Control of peripheral ILC plasticity by transcription factors}

'Plasticity' describes how differentiated cells can acquire new characteristics (phenotype, function) dependent on the environment. This phenomenon has been observed for various cell types in different tissues and has important implications for regenerative medicine. Such plasticity could provide multiple options for immune defense depending on the invading pathogen. ILC functional plasticity has been demonstrated in both human and mouse systems, especially for an ILC3 to ILC1 transition that resembles the well-characterized $T_{H} 17$ to $T_{H} 1$ transition (reviewed in ${ }^{139}$ ). Transcription factors such as $\mathrm{ROR} \gamma \mathrm{t}$ and $\mathrm{T}$-bet play a central role in this process. Loss of ROR $\gamma \mathrm{t}$ and acquisition of T-bet expression is associated with the ILC3 to ILC1 transition that generates 'ex-ILC3' cells that lose IL-22 production and gain the capacity to secrete IFN $\gamma^{51,52}$. Changes in environmental signals may initiate this process. RORyt expression in ILC3 is stabilized by microbiota and IL-7 signals, whereas up-regulation of IL-15 expression could down-regulate ROR $\gamma$ t and enhance T-bet-mediated IFN $\gamma$ expression ${ }^{52}$. This ILC3 to ILC1 transition seems to be crucial for immune defense against Salmonella typhimurium infection ${ }^{51}$. The ILC3 to ILC1 transition in humans is driven by soluble factors, including IL-2 and IL-12 $2^{58,140}$.

Functional plasticity in other ILC subsets is not well documented, although the transcription factor GFI1 may be involved in regulating ILC2 cytokine production profiles. In the absence of GFI1, ILC2s begin to up-regulate ROR $\gamma$ t and produce IL-17 ${ }^{109}$. Whether this occurs in vivo during type 2 immune responses is not known. Phenotypic plasticity of EOMES ${ }^{+}$ILC1s has also been observed ${ }^{8}$ 
although the functional consequences are not known. The fact that ILC plasticity has been predominantly observed in the small intestine suggests that the environment might exert substantial influence on cell-fate decisions, which could be interpreted as tissue imprinting. A role for epigenetic modifications in this process is likely, but this has not yet been established. It will be interesting to explore in future studies whether ILC plasticity has a role in disease progression during infection or inflammation.

\section{CONCLUDING REMARKS}

The discovery of diverse ILC subsets represents a major advance in our understanding of how immune responses are established in order to cope with infection, inflammation and tissue repair. Interestingly, ILCs occupy niches in close vicinity to commensal microorganisms in the gastrointestinal, respiratory, urogenital tract and the skin, where ILCs are responsive to signals emanating from barrier cells (epithelial cells) or from sentinels (myeloid cells). Under steady-state conditions, these signals might help to maintain tissue ILCs. By contrast, during infection or inflammation, different signals are likely to activate ILCs in order to overcome microbial threats but with potentially negative effects on microbial communities (dysbiosis). The overall ILC response may be beneficial to the host in helping to eradicate pathogens, but could also be detrimental; for example, the inability to return to homeostasis could contribute to chronic inflammation and diseases such as IBD, obesity or type 2 diabetes. Deciphering the signals that promote ILC subset development and regulate their peripheral function will be key to understanding the balance between these outcomes. 


\section{Box1 | On the distinction between 'killer' versus 'helper' ILC subsets}

For adaptive immunity, the distinction between cytokine-producing 'helper' $\mathrm{CD}^{+}{ }^{+} \mathrm{T}$ cells and 'killer' $\mathrm{CD}^{+} \mathrm{T}$ cells (cytotoxic $\mathrm{T}$ lymphocytes or CTLs) derives from models of 'cellular' versus 'humoral' immunity. While the developmental pathways of $\mathrm{CD}^{+}$and $\mathrm{CD}^{+} \mathrm{T}$ cell subsets are separate, the functional outputs of these two cell types are overlapping. Cytotoxicity is not a unique property of $\mathrm{CD} 8^{+}$ CTLs ('killer' $\mathrm{CD} 4^{+} \mathrm{CTL}$ exist ${ }^{141}$ ), whereas $\mathrm{CD}^{+} \mathrm{T}$ cells are potent cytokine producers that 'help' immune responses through cellular recruitment and enhanced antigen presentation. Recently the notion of 'helper' versus 'killer' ILCs has surfaced, based on the following evidence: i) Natural killer (NK) cells, but not other ILC subsets, harbour cytotoxic granules; ii) $\mathrm{CD}_{127^{-}} \mathrm{NK}$ cells are independent of GATA3, which is essential for development of all other CD127 $7^{+}$ILC subsets; and iii) committed ILC precursors generate multiple ILC subsets in vivo and in vitro, but poorly generate NK cells under these conditions. While the last point will require additional study to confirm or refute, the fact that NK cells have unique functional characteristics and transcription factor requirements is not sufficient in our opinion to merit their separate classification as 'killer' ILC versus other 'helper' ILCs. NK cells are clearly ILCs being ID2-dependent and CLP-derived. NK cells are an important innate source of cytokines that 'help' immune responses. Although NK cells are cytotoxic, the cytotoxic potentials of other ILC subsets is not fully appreciated. EOMES ${ }^{-}$ILC1 subsets express TRAIL and secrete TNF that can induce apoptosis in susceptible target cells. Other ILC subsets may secrete factors that induce cell death or recruit myeloid cells or macrophages that eliminate targets. Under these circumstances, couldn't these ILCs be considered as 'killers'? Finally, ILC subsets have overlapping transcription factor requirements that transcend the 'killer' versus 'helper' classification. Ablation of TOX eliminates lymphoid tissue-inducing (LTi) cells (a subset of ILC3s) that have no known 'killer' activity as well as cytotoxic NK cells. Could NK and LTi cells have common (and biologically important) functional properties that merit their classification together as $\mathrm{TOX}^{+}$ILC that are separate from other ILCs? 


\section{Box 2 | iCD8 $\alpha \alpha$ cells: to be or not to be an ILC?}

Common lymphoid progenitor (CLP)-derived innate lymphoid cells (ILCs) represent an important effector population for innate immune responses. Do other ILC subsets exist? Recently, OliverasVillagomez group's identified and characterized a new innate cell population characterized by expression of CD8 $\alpha$ homodimers, which they called 'iCD8 $\alpha \alpha$ cells' $^{142}$. Localized in the intestinal epithelium, iCD8 $\alpha \alpha$ cells lack CD127 expression (unlike most ILCs) and instead express a constellation of markers (CD11b, CD11c, CD16/32, CD103, Lag-3 and the MHC class Ib protein H2T3 in mice) that are not routinely found on ILCs. Nevertheless, iCD $8 \alpha \alpha$ cells are derived from CLPs and show evidence for previous Rag expression (ie: fate-mapped in Rag1-Cre reporter mice) similar to that recently shown for NK cells and ILC ${ }^{143}$. The relationship of iCD $8 \alpha \alpha$ cells to NK cells is also evident by their expression of CD122 (IL-2R $\beta$ ) and their dependence on IL-15 for normal development. However, iCD8 $\alpha \alpha$ cells develop independently of ID2, which is an essential orchestrator of ILC differentiation. Taken together, iCD $8 \alpha \alpha$ cells appear to defy any simple classification into known hematopoietic lineages. Interestingly, iCD $8 \alpha \alpha^{+}$cells appear to be involved in bacterial defense and express granzymes and IFN $\gamma$ after IL-12-mediated activation. iCD $8 \alpha \alpha^{+}$cells also interact with the others immune cells by secretion of CCL2, CCL4, CCL5 and CXCL2 and demonstrate antigen presentation capacity after phagocytosis. More work will be required in order to understand the developmental relationships of iCD $8 \alpha \alpha$ cells to other hematopoietic cells and their place within innate immune defense. 
Box $3 \mid$ The evolutionary relationship between ILC subsets and $T_{H}$ cells.

Who came first (evolutionarily speaking): innate lymphoid cells (ILCs) or T helper ( $\left.T_{H}\right)$ cells? One can imagine that pressure to develop diversified defenses evolved as the microbial world expanded. In addition to cellular mechanisms of phagocytosis, more sophisticated systems involving cellular barriers, soluble anti-microbial factors and diverse effector cells were needed. This necessitated coordination between different cell types that likely led to well-characterized present day 'type $X$ ' responses that combat viruses, intra- and extra-cellular bacteria, fungi and parasites. Prior to the emergence of antigen receptors, ILCs would have evolved to be part of this new defense system. The fitness advantage afforded by diversified ILC groups would be two-fold: first, ILCs could act to relay primary events of infection to the appropriate effector cells; in this way ILCs (like sentinel cells) interpret immunity. Second, ILCs would act to amplify the response due to robust cytokine production and probably through cellular proliferation. With the advent of TCRs, adaptive $T_{H}$ cells would co-opt this 'blueprint' and embellish it with additional features, including fine antigen specificity, clonal expansion and long-lived immunological memory. As such, the idea that ILCs were precursors to $T_{H}$ cells makes sense. However, ILCs are developmentally dependent on ID2-mediated repression of $E$ protein-driven B and T cell development. If ILCs evolved first, then how can one explain the selective pressure to develop ID2 to counteract B and T cell pathways that don't exist? One possibility is that ILCs may have initially needed ID2 (and other IDs) for another reason: to diverge from primitive proliferating hematopoietic stem cells (that express high $E$ protein levels). Further studies in invertebrate species will hopefully provide the answers to these questions. 


\section{References}

1. Spits, H. \& Di Santo, J. P. The expanding family of innate lymphoid cells: regulators and effectors of immunity and tissue remodeling. Nat Immunol 12, 21-27 (2011).

2. Spits, H. et al. Innate lymphoid cells - a proposal for uniform nomenclature. Nat. Rev. Immunol. 1-5 (1).

3. Di Santo, J. P. Natural killer cell developmental pathways: a question of balance. Annu. Rev. Immunol. 24, 257-286 (2006).

4. Shi, F. D., Ljunggren, H. G., La Cava, A. \& Van Kaer, L. Organ-specific features of natural killer cells. Nat Rev Immunol 11, 658-671 (2011).

5. Tay, C. H. \& Welsh, R. M. Distinct organ-dependent mechanisms for the control of murine cytomegalovirus infection by natural killer cells. J. Virol. 71, 267-275 (1997).

6. Cooper, M. A., Fehniger, T. A. \& Caligiuri, M. A. The biology of human natural killercell subsets. Trends Immunol 22, 633-640 (2001).

7. Fuchs, A. et al. Intraepithelial Type 1 Innate Lymphoid Cells Are a Unique Subset of IL-12- and IL-15-Responsive IFN-\&gamma;-Producing Cells. Immunity 38, 769-781 (2013).

8. Klose, C. S. et al. Differentiation of Type 1 ILCs from a Common Progenitor to All Helper-like Innate Lymphoid Cell Lineages. Cell 157, 340-356 (2014).

9. Diefenbach, A., Colonna, M. \& Koyasu, S. Development, Differentiation, and Diversity of Innate Lymphoid Cells. Immunity 41, 354-365 (2014).

10. Daussy, C. et al. T-bet and Eomes instruct the development of two distinct natural killer cell lineages in the liver and in the bone marrow. J Exp Med 211, 563-577 (2014).

11. Marquardt, N. et al. Cutting Edge: Identification and Characterization of Human Intrahepatic CD49a+ NK Cells. J. Immunol. Baltim. Md 1950 (2015). doi:10.4049/jimmunol.1402756

12. Bernink, J. H. et al. Human type 1 innate lymphoid cells accumulate in inflamed mucosal tissues. Nat Immunol 14, 221-229 (2013).

13. Moro, K. et al. Innate production of $\mathrm{T}(\mathrm{H}) 2$ cytokines by adipose tissue-associated cKit(+)Sca-1(+) lymphoid cells. Nature 463, 540-544 (2010).

14. Neill, D. R. et al. Nuocytes represent a new innate effector leukocyte that mediates type-2 immunity. Nature 464, 1367-1370 (2010).

15. Price, A. E. et al. Systemically dispersed innate IL-13-expressing cells in type 2 immunity. Proc Natl Acad Sci U A 107, 11489-11494 (2010).

16. Halim, T. Y., Krauss, R. H., Sun, A. C. \& Takei, F. Lung natural helper cells are a critical source of Th2 cell-type cytokines in protease allergen-induced airway inflammation. Immunity 36, 451-463 (2012).

17. Ikutani, M. et al. Identification of innate IL-5-producing cells and their role in lung eosinophil regulation and antitumor immunity. J. Immunol. Baltim. Md 1950 188, 703713 (2012).

18. Fallon, P. G. et al. Identification of an interleukin (IL)-25-dependent cell population that provides IL-4, IL-5, and IL-13 at the onset of helminth expulsion. $J$ Exp Med 203, 1105-1116 (2006).

19. Bartemes, K. R. et al. IL-33-responsive lineage- CD25+ CD44(hi) lymphoid cells mediate innate type 2 immunity and allergic inflammation in the lungs. J Immunol 188, 1503-1513 (2012).

20. Chang, Y. J. et al. Innate lymphoid cells mediate influenza-induced airway hyperreactivity independently of adaptive immunity. Nat Immunol 12, 631-638 (2011).

21. Monticelli, L. A. et al. Innate lymphoid cells promote lung-tissue homeostasis after infection with influenza virus. Nat Immunol 12, 1045-1054 (2011).

22. Molofsky, A. B. et al. Innate lymphoid type 2 cells sustain visceral adipose tissue 
eosinophils and alternatively activated macrophages. $J$ Exp Med 210, 535-549 (2013).

23. Klein Wolterink, R. G. et al. Essential, dose-dependent role for the transcription factor Gata3 in the development of IL-5+ and IL-13+ type 2 innate lymphoid cells. Proc Natl Acad Sci U A 110, 10240-10245 (2013).

24. Roediger, B. et al. Cutaneous immunosurveillance and regulation of inflammation by group 2 innate lymphoid cells. Nat Immunol 14, 564-573 (2013).

25. Wilhelm, C. et al. An IL-9 fate reporter demonstrates the induction of an innate IL-9 response in lung inflammation. Nat Immunol 12, 1071-1077 (2011).

26. Barlow, J. L. et al. Innate IL-13-producing nuocytes arise during allergic lung inflammation and contribute to airways hyperreactivity. J Allergy Clin Immunol 129, 191-194 (2012).

27. Salimi, M. et al. A role for IL-25 and IL-33-driven type-2 innate lymphoid cells in atopic dermatitis. J. Exp. Med. 210, 2939-2950 (2013).

28. Hams, E. et al. IL-25 and type 2 innate lymphoid cells induce pulmonary fibrosis. Proc. Natl. Acad. Sci. U. S. A. 111, 367-372 (2014).

29. McHedlidze, T. et al. Interleukin-33-dependent innate lymphoid cells mediate hepatic fibrosis. Immunity 39, 357-371 (2013).

30. Furusawa, J. et al. Critical role of p38 and GATA3 in natural helper cell function. $J$ Immunol 191, 1818-1826 (2013).

31. Hoyler, T. et al. The Transcription Factor GATA-3 Controls Cell Fate and Maintenance of Type 2 Innate Lymphoid Cells. Immunity 37, 634-648 (2012).

32. Yagi, R. et al. The transcription factor GATA3 is critical for the development of all IL7Ralpha-expressing innate lymphoid cells. Immunity 40, 378-388 (2014).

33. Mjosberg, J. M. et al. Human IL-25- and IL-33-responsive type 2 innate lymphoid cells are defined by expression of CRTH2 and CD161. Nat Immunol 12, 1055-1062 (2011).

34. Liang, H. E. et al. Divergent expression patterns of IL-4 and IL-13 define unique functions in allergic immunity. Nat Immunol 13, 58-66 (2012).

35. Bernink, J. H., Germar, K. \& Spits, H. The role of ILC2 in pathology of type 2 inflammatory diseases. Curr. Opin. Immunol. 31C, 115-120 (2014).

36. Brestoff, J. R. et al. Group 2 innate lymphoid cells promote beiging of white adipose tissue and limit obesity. Nature (2014). doi:10.1038/nature14115

37. Lee, M.-W. et al. Activated Type 2 Innate Lymphoid Cells Regulate Beige Fat Biogenesis. Cell (2014). doi:10.1016/j.cell.2014.12.011

38. Walker, J. A., Barlow, J. L. \& McKenzie, A. N. Innate lymphoid cells-how did we miss them? Nat Rev Immunol 13, 75-87 (2013).

39. Van de Pavert, S. A. \& Mebius, R. E. New insights into the development of lymphoid tissues. Nat Rev Immunol 10, 664-674 (2010).

40. Kanamori, Y. et al. Identification of novel lymphoid tissues in murine intestinal mucosa where clusters of c-kit+ IL-7R+ Thy1+ lympho-hemopoietic progenitors develop. J Exp Med 184, 1449-1459 (1996).

41. Bouskra, D. et al. Lymphoid tissue genesis induced by commensals through NOD1 regulates intestinal homeostasis. Nature 456, 507-510 (2008).

42. Leavy, O. Mucosal immunology: Multifunctional gut $\operatorname{IgA}(+)$ plasma cells. Nat Rev Immunol 12, 75 (2012).

43. Sawa, S. et al. Lineage relationship analysis of RORgammat+ innate lymphoid cells. Science 330, 665-669 (2010).

44. Takatori, H. et al. Lymphoid tissue inducer-like cells are an innate source of IL-17 and IL-22. J Exp Med 206, 35-41 (2009).

45. Eberl, G. et al. An essential function for the nuclear receptor RORgamma(t) in the generation of fetal lymphoid tissue inducer cells. Nat Immunol 5, 64-73 (2004). 
46. Cherrier, M., Sawa, S. \& Eberl, G. Notch, Id2, and RORgammat sequentially orchestrate the fetal development of lymphoid tissue inducer cells. J Exp Med 209, 729740 (2012).

47. Satoh-Takayama, N. et al. Microbial flora drives interleukin 22 production in intestinal NKp46+ cells that provide innate mucosal immune defense. Immunity 29, 958-970 (2008).

48. Sanos, S. L. et al. RORgammat and commensal microflora are required for the differentiation of mucosal interleukin 22-producing NKp46+ cells. Nat Immunol 10, 83-91 (2009).

49. Luci, C. et al. Influence of the transcription factor RORgammat on the development of NKp46+ cell populations in gut and skin. Nat Immunol 10, 75-82 (2009).

50. Buonocore, S. et al. Innate lymphoid cells drive interleukin-23-dependent innate intestinal pathology. Nature 464, 1371-1375 (2010).

51. Klose, C. S. et al. A T-bet gradient controls the fate and function of CCR6RORgammat+ innate lymphoid cells. Nature 494, 261-265 (2013).

52. Vonarbourg, C. et al. Regulated expression of nuclear receptor RORgammat confers distinct functional fates to $\mathrm{NK}$ cell receptor-expressing RORgammat(+) innate lymphocytes. Immunity 33, 736-751 (2010).

53. Hepworth, M. R. et al. Innate lymphoid cells regulate CD4+ T-cell responses to intestinal commensal bacteria. Nature 498, 113-117 (2013).

54. Neurath, M. F. Cytokines in inflammatory bowel disease. Nat. Rev. Immunol. 14, 329342 (2014).

55. Cupedo, T. et al. Human fetal lymphoid tissue-inducer cells are interleukin 17producing precursors to RORC+ CD127+ natural killer-like cells. Nat Immunol 10, 6674 (2009).

56. Sonnenberg, G. F. et al. Innate lymphoid cells promote anatomical containment of lymphoid-resident commensal bacteria. Science 336, 1321-1325 (2012).

57. Hoorweg, K. et al. Functional Differences between Human NKp44(-) and NKp44(+) RORC(+) Innate Lymphoid Cells. Front Immunol 3, 72 (2012).

58. Cella, M. et al. A human natural killer cell subset provides an innate source of IL-22 for mucosal immunity. Nature 457, 722-725 (2009).

59. Geremia, A. et al. IL-23-responsive innate lymphoid cells are increased in inflammatory bowel disease. J Exp Med 208, 1127-1133 (2011).

60. Ng, S. Y.-M., Yoshida, T., Zhang, J. \& Georgopoulos, K. Genome-wide lineagespecific transcriptional networks underscore Ikaros-dependent lymphoid priming in hematopoietic stem cells. Immunity 30, 493-507 (2009).

61. Rothenberg, E. V. Transcriptional control of early T and B cell developmental choices. Annu. Rev. Immunol. 32, 283-321 (2014).

62. Yoshida, H. et al. Expression of alpha(4)beta(7) integrin defines a distinct pathway of lymphoid progenitors committed to $\mathrm{T}$ cells, fetal intestinal lymphotoxin producer, $\mathrm{NK}$, and dendritic cells. J Immunol 167, 2511-2521 (2001).

63. Possot, C. et al. Notch signaling is necessary for adult, but not fetal, development of RORgammat(+) innate lymphoid cells. Nat Immunol 12, 949-958 (2011).

64. Constantinides, M. G., McDonald, B. D., Verhoef, P. A. \& Bendelac, A. A committed precursor to innate lymphoid cells. Nature 508, 397-401 (2014).

65. $\mathrm{Yu}, \mathrm{X}$. et al. The basic leucine zipper transcription factor NFIL3 directs the development of a common innate lymphoid cell precursor. eLife 3, (2014).

66. Rosmaraki, E. E. et al. Identification of committed NK cell progenitors in adult murine bone marrow. Eur. J. Immunol. 31, 1900-1909 (2001).

67. Carotta, S., Pang, S. H. M., Nutt, S. L. \& Belz, G. T. Identification of the earliest NK- 
cell precursor in the mouse BM. Blood 117, 5449-5452 (2011).

68. Fathman, J. W. et al. Identification of the earliest natural killer cell-committed progenitor in murine bone marrow. Blood 118, 5439-5447 (2011).

69. Verykokakis, M., Zook, E. C. \& Kee, B. L. ID'ing innate and innate-like lymphoid cells. Immunol. Rev. 261, 177-197 (2014).

70. Boos, M. D., Yokota, Y., Eberl, G. \& Kee, B. L. Mature natural killer cell and lymphoid tissue-inducing cell development requires Id2-mediated suppression of $\mathrm{E}$ protein activity. J Exp Med 204, 1119-1130 (2007).

71. Yokota, Y. et al. Development of peripheral lymphoid organs and natural killer cells depends on the helix-loop-helix inhibitor Id2. Nature 397, 702-706 (1999).

72. Ikawa, T., Fujimoto, S., Kawamoto, H., Katsura, Y. \& Yokota, Y. Commitment to natural killer cells requires the helix-loop-helix inhibitor Id2. Proc Natl Acad Sci U A 98, 5164-5169 (2001).

73. $\mathrm{Xu}, \mathrm{W}$. et al. E2A transcription factors limit expression of Gata3 to facilitate $\mathrm{T}$ lymphocyte lineage commitment. Blood 121, 1534-1542 (2013).

74. Kwon, K. et al. Instructive role of the transcription factor E2A in early B lymphopoiesis and germinal center B cell development. Immunity 28, 751-762 (2008).

75. Kee, B. L. \& Murre, C. Induction of early B cell factor (EBF) and multiple B lineage genes by the basic helix-loop-helix transcription factor E12. J. Exp. Med. 188, 699-713 (1998).

76. Heemskerk, M. H. et al. Inhibition of $\mathrm{T}$ cell and promotion of natural killer cell development by the dominant negative helix loop helix factor Id3. J. Exp. Med. 186, 1597-1602 (1997).

77. Nakahiro, T., Kurooka, H., Mori, K., Sano, K. \& Yokota, Y. Identification of BMPresponsive elements in the mouse Id 2 gene. Biochem. Biophys. Res. Commun. 399, 416-421 (2010).

78. Tong, X. et al. Transcriptional repressor E4-binding protein 4 (E4BP4) regulates metabolic hormone fibroblast growth factor 21 (FGF21) during circadian cycles and feeding. J. Biol. Chem. 285, 36401-36409 (2010).

79. Yamajuku, D. et al. Cellular DBP and E4BP4 proteins are critical for determining the period length of the circadian oscillator. FEBS Lett. 585, 2217-2222 (2011).

80. Male, V., Nisoli, I., Gascoyne, D. M. \& Brady, H. J. M. E4BP4: an unexpected player in the immune response. Trends Immunol. 33, 98-102 (2012).

81. Gascoyne, D. M. et al. The basic leucine zipper transcription factor E4BP4 is essential for natural killer cell development. Nat Immunol 10, 1118-1124 (2009).

82. Kamizono, S. et al. Nfil3/E4bp4 is required for the development and maturation of NK cells in vivo. J Exp Med 206, 2977-2986 (2009).

83. Geiger, T. L. et al. Nfil3 is crucial for development of innate lymphoid cells and host protection against intestinal pathogens. J Exp Med 211, 1723-1731 (2014).

84. Seillet, C. et al. Nfil3 is required for the development of all innate lymphoid cell subsets. $J$ Exp Med 211, 1733-1740 (2014).

85. $\mathrm{Xu}, \mathrm{W}$. et al. NFIL3 orchestrates the emergence of common helper innate lymphoid cell precursors. Cell Reports in press (2015).

86. Ikushima, S. et al. Pivotal role for the NFIL3/E4BP4 transcription factor in interleukin 3-mediated survival of pro-B lymphocytes. Proc. Natl. Acad. Sci. U. S. A. 94, 26092614 (1997).

87. Seillet, C. et al. Differential requirement for Nfil3 during NK cell development. $J$ Immunol 192, 2667-2676 (2014).

88. Male, V. et al. The transcription factor E4bp4/Nfil3 controls commitment to the NK lineage and directly regulates Eomes and Id2 expression. J Exp Med 211, 635-642 
(2014).

89. Tindemans, I., Serafini, N., Di Santo, J. P. \& Hendriks, R. W. GATA-3 Function in Innate and Adaptive Immunity. Immunity 41, 191-206 (2014).

90. Vosshenrich, C. A. et al. A thymic pathway of mouse natural killer cell development characterized by expression of GATA-3 and CD127. Nat Immunol 7, 1217-1224 (2006).

91. Serafini, N. et al. Gata3 drives development of RORgammat+ group 3 innate lymphoid cells. J Exp Med 211, 199-208 (2014).

92. Samson, S. I. et al. GATA-3 promotes maturation, IFN-gamma production, and liverspecific homing of NK cells. Immunity 19, 701-711 (2003).

93. Constantinides, M. G. \& Bendelac, A. Transcriptional regulation of the NKT cell lineage. Curr. Opin. Immunol. 25, 161-167 (2013).

94. Murphy, K. M. \& Reiner, S. L. The lineage decisions of helper T cells. Nat. Rev. Immunol. 2, 933-944 (2002).

95. Colucci, F. et al. Natural cytotoxicity uncoupled from the Syk and ZAP-70 intracellular kinases. Nat. Immunol. 3, 288-294 (2002).

96. Magram, J. et al. IL-12-deficient mice are defective in IFN gamma production and type 1 cytokine responses. Immunity 4, 471-481 (1996).

97. Aliahmad, P., de la Torre, B. \& Kaye, J. Shared dependence on the DNA-binding factor TOX for the development of lymphoid tissue-inducer cell and NK cell lineages. Nat Immunol 11, 945-952 (2010).

98. Gordon, S. M. et al. The transcription factors T-bet and Eomes control key checkpoints of natural killer cell maturation. Immunity 36, 55-67 (2012).

99. Barton, K. et al. The Ets-1 transcription factor is required for the development of natural killer cells in mice. Immunity 9, 555-563 (1998).

100. Djuretic, I. M. et al. Transcription factors T-bet and Runx3 cooperate to activate Ifng and silence I14 in T helper type 1 cells. Nat. Immunol. 8, 145-153 (2007).

101. Vahedi, G. et al. Helper T-cell identity and evolution of differential transcriptomes and epigenomes. Immunol. Rev. 252, 24-40 (2013).

102. Aliahmad, P. \& Kaye, J. Development of all CD4 T lineages requires nuclear factor TOX. J. Exp. Med. 205, 245-256 (2008).

103. Wilkinson, B. et al. TOX: an HMG box protein implicated in the regulation of thymocyte selection. Nat. Immunol. 3, 272-280 (2002).

104. Sharrocks, A. D. The ETS-domain transcription factor family. Nat. Rev. Mol. Cell Biol. 2, 827-837 (2001).

105. Muthusamy, N., Barton, K. \& Leiden, J. M. Defective activation and survival of T cells lacking the Ets-1 transcription factor. Nature 377, 639-642 (1995).

106. Bories, J. C. et al. Increased T-cell apoptosis and terminal B-cell differentiation induced by inactivation of the Ets-1 proto-oncogene. Nature 377, 635-638 (1995).

107. Ramirez, K. et al. Gene deregulation and chronic activation in natural killer cells deficient in the transcription factor ETS1. Immunity 36, 921-932 (2012).

108. Wong, S. H. et al. Transcription factor RORalpha is critical for nuocyte development. Nat Immunol 13, 229-236 (2012).

109. Spooner, C. J. et al. Specification of type 2 innate lymphocytes by the transcriptional determinant Gfi1. Nat. Immunol. 14, 1229-1236 (2013).

110. Yang, Q. et al. T cell factor 1 is required for group 2 innate lymphoid cell generation. Immunity 38, 694-704 (2013).

111. Huang, Y. et al. IL-25-responsive, lineage-negative KLRG1(hi) cells are multipotential 'inflammatory' type 2 innate lymphoid cells. Nat. Immunol. (2014). doi:10.1038/ni.3078

112. Koyasu, S. Inflammatory ILC2 cells: disguising themselves as progenitors? Nat. 
Immunol. 16, 133-134 (2015).

113. Mielke, L. A. et al. TCF-1 Controls ILC2 and NKp46+RORgammat+ Innate Lymphocyte Differentiation and Protection in Intestinal Inflammation. J Immunol 191, 4383-4391 (2013).

114. Hamilton, B. A. et al. Disruption of the nuclear hormone receptor RORalpha in staggerer mice. Nature 379, 736-739 (1996).

115. Halim, T. Y. et al. Retinoic-acid-receptor-related orphan nuclear receptor alpha is required for natural helper cell development and allergic inflammation. Immunity 37, 463-474 (2012).

116. Montaldo, E. et al. Human ROR $\gamma \mathrm{t}(+) \mathrm{CD} 34(+)$ Cells Are Lineage-Specified Progenitors of Group 3 ROR $\gamma \mathrm{t}(+)$ Innate Lymphoid Cells. Immunity 41, 988-1000 (2014).

117. Lee, J. S. et al. AHR drives the development of gut ILC22 cells and postnatal lymphoid tissues via pathways dependent on and independent of Notch. Nat Immunol 13, 144-151 (2012).

118. Kiss, E. A. et al. Natural aryl hydrocarbon receptor ligands control organogenesis of intestinal lymphoid follicles. Science 334, 1561-1565 (2011).

119. Qiu, J. et al. The aryl hydrocarbon receptor regulates gut immunity through modulation of innate lymphoid cells. Immunity 36, 92-104 (2012).

120. Van de Pavert, S. A. et al. Maternal retinoids control type 3 innate lymphoid cells and set the offspring immunity. Nature 508, 123-127 (2014).

121. Spencer, S. P. et al. Adaptation of innate lymphoid cells to a micronutrient deficiency promotes type 2 barrier immunity. Science 343, 432-437 (2014).

122. Rankin, L. C. et al. The transcription factor T-bet is essential for the development of NKp46+ innate lymphocytes via the Notch pathway. Nat Immunol 14, 389-395 (2013).

123. Sciume, G. et al. Distinct requirements for T-bet in gut innate lymphoid cells. $J$ Exp Med 209, 2331-2338 (2012).

124. Yoshida, H. et al. IL-7 receptor alpha+ CD3(-) cells in the embryonic intestine induces the organizing center of Peyer's patches. Int Immunol 11, 643-655 (1999).

125. Bando, J. K., Liang, H.-E. \& Locksley, R. M. Identification and distribution of developing innate lymphoid cells in the fetal mouse intestine. Nat. Immunol. (2014). doi: $10.1038 /$ ni.3057

126. Vosshenrich, C. A. J. et al. Roles for common cytokine receptor gamma-chaindependent cytokines in the generation, differentiation, and maturation of NK cell precursors and peripheral NK cells in vivo. J. Immunol. Baltim. Md 1950 174, 1213 1221 (2005).

127. Freud, A. G. et al. A human CD34(+) subset resides in lymph nodes and differentiates into CD56bright natural killer cells. Immunity 22, 295-304 (2005).

128. Puzanov, I. J., Bennett, M. \& Kumar, V. IL-15 can substitute for the marrow microenvironment in the differentiation of natural killer cells. J. Immunol. Baltim. Md 1950 157, 4282-4285 (1996).

129. Kennedy, M. K. et al. Reversible defects in natural killer and memory CD8 T cell lineages in interleukin 15-deficient mice. J. Exp. Med. 191, 771-780 (2000).

130. Satoh-Takayama, N. et al. IL-7 and IL-15 independently program the differentiation of intestinal CD3-NKp46+ cell subsets from Id2-dependent precursors. J Exp Med 207, 273-280 (2010).

131. Huntington, N. D. et al. IL-15 trans-presentation promotes human NK cell development and differentiation in vivo. J Exp Med 206, 25-34 (2009).

132. Schmutz, S. et al. Cutting edge: IL-7 regulates the peripheral pool of adult ROR gamma+ lymphoid tissue inducer cells. J. Immunol. Baltim. Md 1950 183, 2217-2221 (2009). 
133. Merzoug, L. B. et al. Conditional ablation of NKp46+ cells using a novel Ncrl(greenCre) mouse strain: NK cells are essential for protection against pulmonary B16 metastases. Eur. J. Immunol. 44, 3380-3391 (2014).

134. Ranson, T. et al. IL-15 is an essential mediator of peripheral NK-cell homeostasis. Blood 101, 4887-4893 (2003).

135. Lodolce, J. P. et al. IL-15 receptor maintains lymphoid homeostasis by supporting lymphocyte homing and proliferation. Immunity 9, 669-676 (1998).

136. Liou, Y.-H. et al. Adipocyte IL-15 regulates local and systemic NK cell development. $J$. Immunol. Baltim. Md 1950 193, 1747-1758 (2014).

137. Leavy, O. Natural killer cells: adaptive control of NK cells. Nat. Rev. Immunol. 13, 394 (2013).

138. Sun, J. C., Beilke, J. N. \& Lanier, L. L. Adaptive immune features of natural killer cells. Nature 457, 557-561 (2009).

139. Kanno, Y., Vahedi, G., Hirahara, K., Singleton, K. \& O’Shea, J. J. Transcriptional and epigenetic control of $\mathrm{T}$ helper cell specification: molecular mechanisms underlying commitment and plasticity. Annu. Rev. Immunol. 30, 707-731 (2012).

140. Hughes, T. et al. Interleukin-1beta selectively expands and sustains interleukin-22+ immature human natural killer cells in secondary lymphoid tissue. Immunity 32, 803 814 (2010).

141. Van de Berg, P. J., van Leeuwen, E. M., ten Berge, I. J. \& van Lier, R. Cytotoxic human CD4(+) T cells. Curr. Opin. Immunol. 20, 339-343 (2008).

142. Van Kaer, L. et al. CD8 $\alpha \alpha+$ Innate-Type Lymphocytes in the Intestinal Epithelium Mediate Mucosal Immunity. Immunity 41, 451-464 (2014).

143. Karo, J. M., Schatz, D. G. \& Sun, J. C. The RAG recombinase dictates functional heterogeneity and cellular fitness in natural killer cells. Cell 159, 94-107 (2014). 


\section{FIGURE LEGENDS}

\section{Figure 1. ILC groups and subsets}

Characterization of mouse and human ILC subsets by surface markers, transcription factors and cytokines. CCR, CC-chemokine receptor; CXCR, CXC-chemokine receptor, IFN, interferon; TNF, tumor necrosis factor; IL, interleukin; IL-R, interleukin receptor; KIR, killer cell immunoglobulin-like receptor; NK, natural killer cell; ILC, innate lymphoid cell; ICOS, inducible T cell co-stimulator; CRTH2, chemoattractant receptor-homologous molecule expressed on $T_{H} 2$ cell; LT, lymphotoxin; GM-CSF, granulocyte-macrophage colony-stimulating factor; ROR $\gamma$ t, RAR-related orphan receptor gamma; GATA3, GATA binding protein 3; TRAIL, TNF-related apoptosis-inducing ligand ; KIR, killer cell Ig-like receptor.

\section{Figure 2. Model for murine ILC development}

Innate lymphoid cells (ILCs) differentiate from hematopoietic stem cell via a common lymphoid progenitor (CLP). Interleukin (IL)-7 and the transcription factor (TF) Id2 and Nfil3 promote the differentiation of potential common precursor of restricted-ILC progenitor (CILCP). Downstream of the CLP, NK precursor (NKP), PLZF ${ }^{-}$and $\mathrm{PLZF}^{+} \mathrm{Id}^{+}$common helper innate lymphoid progenitor (CHILP) can be discriminated and generate different branch of ILC family. The relationship of each precursor remains unclear (see Figure 3). Immature NK cells (iNK) appear after expression of EOMES, TOX and ETS1 in NK precursor (NKP) and mature (mNK) under the influence of T-BET and RUNX3. ILC1 may derive from NKP and/or CHILP via IL-15 via specific transcriptional program that include T-BET, EOMES, GATA3 and/or NFIL3. ILC2 and ILC3 differentiate from the Id ${ }^{+}$CHILP under the influence of specific TFs, IL-7 and retinoic acid (RA). Interestingly, environmental factors, and notably the commensal flora, influence the stability of some TFs, such as ROR $\gamma$.

\section{Figure 3. Models for ILC lineage commitment}

a. Progressive differentiation: common ILC precursors (CILCP) progressively generate ILC subsets during their differentiation and the acquisition of specific transcription factor. In this model, all ILC are generated through a unique pathway. b. Distinct lineages: CLP generate CXCR6 ${ }^{+}$ILC restricted progenitors $(\alpha \mathrm{LP})$ that segregate differentiate into two main ILC lineages. The relative dependency for

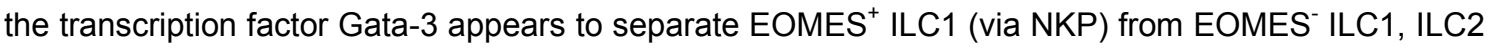
and ILC3 (via CHILP).

\section{Figure 4. ILC maintenance and plasticity in tissues}

Hematopoietic (myeloid and T cells) and non-hematopoietic cells (stromal, endothelial and epithelial cells) provide $\gamma$ c cytokines (IL-2, IL-7, IL-15) that promote the ILC maintenance and proliferation in peripheral tissues. Environmental signals can modulate transcriptional profiles in ILCs and generate ILC functional plasticity, notably for an ILC3 to ILC1 transition. 


\section{ACKNOWLEDGMENTS}

The authors would like to thank all members of the Di Santo lab for helpful discussions. This work is supported by grants from the Institut Pasteur, Inserm, Ligue National Contre le Cancer, and the Agence National pour la Recherche. We apologize to those colleagues whose work has not been cited due to space constraints.

\section{COMPETING INTERESTS STATEMENT}

James Di Santo is a founder and stakeholder in AXENIS (Paris, France).

\section{AUTHOR INFORMATION}

Nicolas Serafini

Nicolas Serafini received a Ph.D. in immunology from the University of Paris VII, France, in the laboratory of Renato Monteiro, where he worked on the role of calcium homeostasis in myeloid cells biology during bacterial infection. He is a postdoctoral fellow in the Innate Immunity Unit studying the development and function of murine ILCs.

\section{Christian A. J. Vosshenrich}

Christian Vosshenrich a permanent staff scientist in the Immunology Department of the Institut Pasteur, where he leads a team in the Innate Immunity Unit. His main research interests involve development of natural killer cells and other innate lymphocytes.

\section{James P. Di Santo}

James Di Santo received a combined M.D./Ph.D. from Cornell University Medical College, New York, USA, and the Memorial Sloan-Kettering Cancer Center, New York, USA. He completed postdoctoral training in Paris, before becoming a permanent staff scientist with the Institut National de la Santé et de la Recherche Médicale (INSERM) in 1994. He currently directs a Research Unit in the Immunology Department at the Institut Pasteur focused on development and function of innate lymphocytes. 


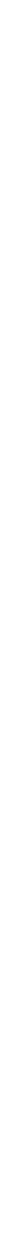




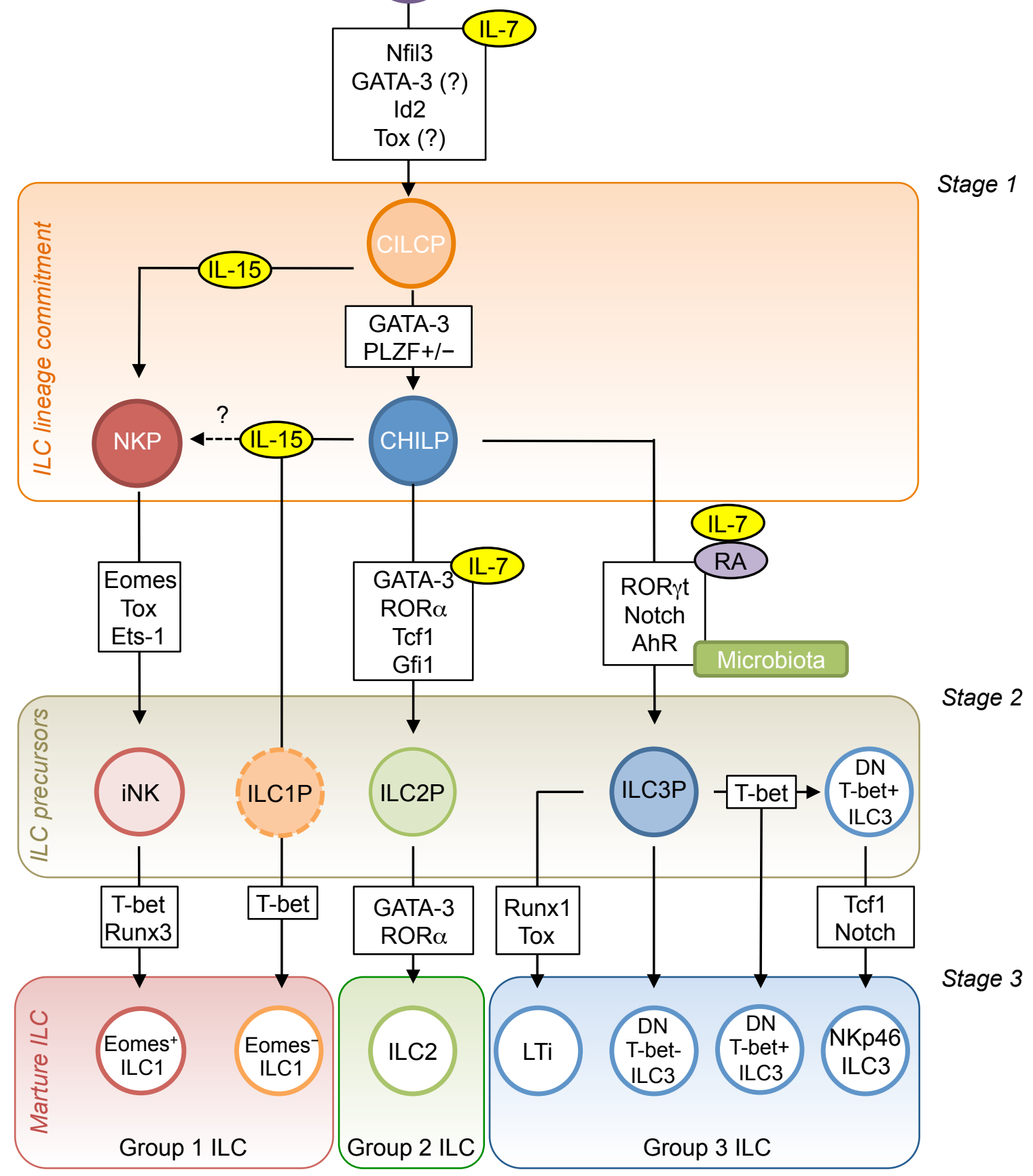




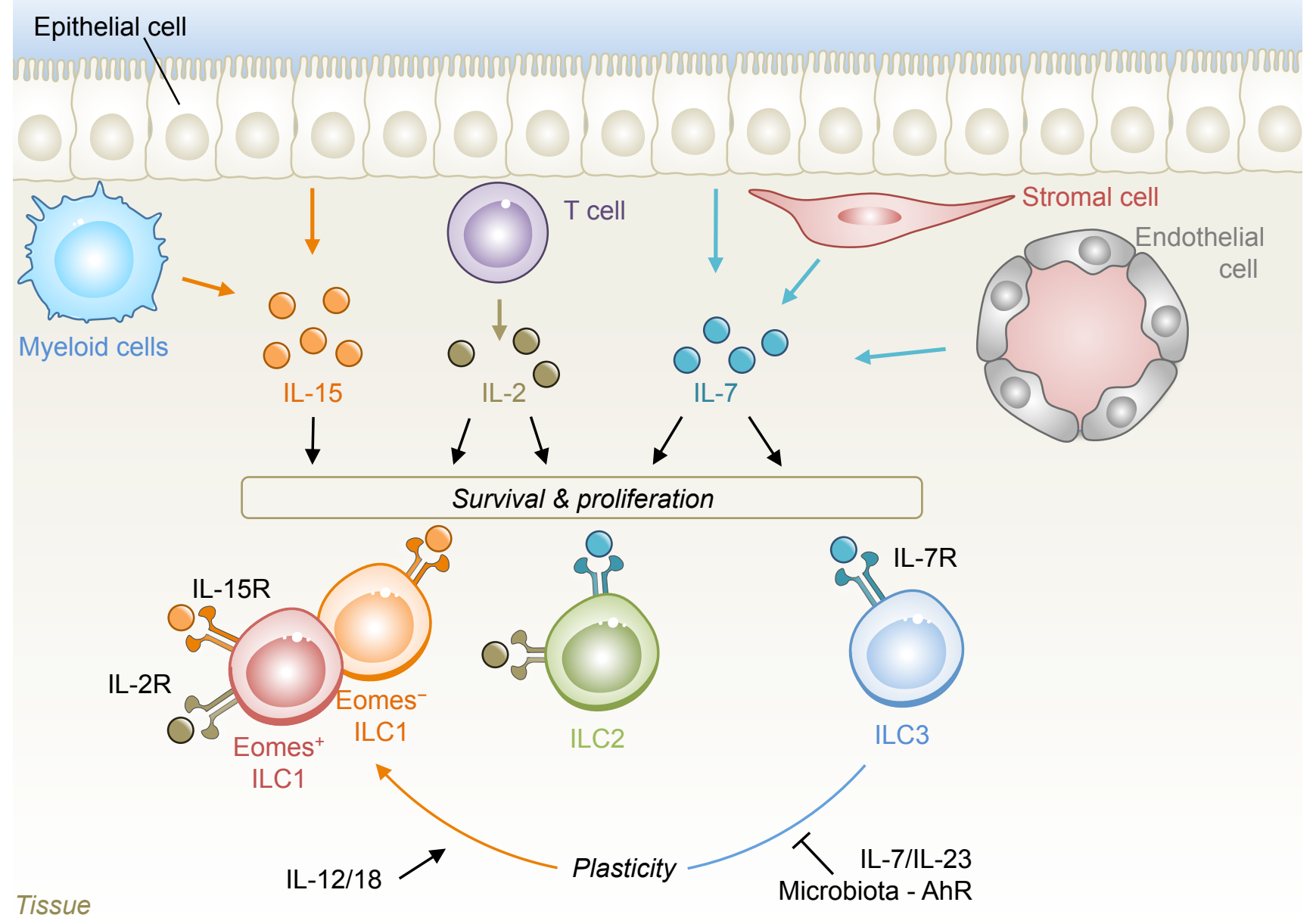

